\title{
Selective indications for the use of praziquantel in the treatment of brain cysticercosis
}

\author{
DANIEL VASCONCELOS,* HÉCTOR CRUZ-SEGURA,* \\ HUMBERTO MATEOS-GOMEZ, $†$ GENARO ZENTENO ALANIS $\dagger$
}

From the Departments of Neurology* and Neurosurgery, $\dagger$ General Hospital, Medical Center of the Mexican

Institute for Social Security, Mexico City, Mexico

SUMMARY The selective use of praziquantel for the treatment of brain cysticercosis in human beings is described, based upon the results of clinical tests conducted during a 2 year period. Forty patients with clinical diagnosis of cysticercosis, confirmed and documented by CT, were treated. They are divided into four groups, representative of the various forms of the disease. The results assessed by clinical evaluation and sequential $\mathrm{CT}$, give a tenpoint criterion of the indications for praziquantel.

The first publications reporting on the treatment of neurocysticercosis with praziquantel appeared in $1980 .^{1-4}$ Because there had previously been no effective treatment for the condition, the positive results initially observed gave rise to new studies which have confirmed the action of praziquantel on cysticerci. ${ }^{5-10}$

The earlier enthusiasm led to inadequate selection of patients. Owing to the peculiarities of cysticercosis, it is not always possible for the drug to influence the disease. Hence it has become necessary to establish in which cases the drug would really be useful.

Following extensive clinical trials conducted during 1982 and 1983 at the Neurology Service, General Hospital, National Medical Center, Mexican Institute for Social Security, Mexico City, 10 criteria are proposed as indications for treating brain cysticercosis with praziquantel in human beings.

\section{Material and methods}

Forty patients with clinically diagnosed cysticercosis, all confirmed and documented by CT, were selected for the trial. Criteria regarding selective indications for such treatment were all based on the serial CT scans, since this is the method par excellence for identifying the parasite characteristics both before and after treatment, as well as for visualising morphological changes caused in the brain by their

Address for reprint requests: Dr Daniel Vasconcelos, Ave. Baja California 180-302 México 7, D. F. cp 06760, Mexico.

Received 7 March 1986 and in revised form 9 July 1986. Accepted 26 July 1986 presence. Moreover, both diagnostic criteria and findings related to therapy responses could also be detected by using CT.

The selected patients were divided into four groups, according to the various modalities of cysticercosis commonly observed by CT. The division was based on the following criteria:

Group 1 patients with cysticercosis cysts disseminated in the brain parenchyma and/or located in the subarachnoid spaces, with no history of neurosurgical operations for their illness; 25 cases.

Group 2 patients with cysts in the brain or subarachnoid spaces, who underwent neurosurgical interventions due to complications of their disease before receiving praziquantel; six cases.

Group 3 patients in whom, by using an original procedure developed at the Neurology Service, were shown to have only intraventricular cysticercous cysts; five cases.

Group 4 patients in whom the CT appearances gave rise to the denomination of "milliary" cysticercosis; four cases.

All patients were hospitalised throughout the duration of treatment. Each patient underwent neurological examination, CT, lumbar puncture, cerebrospinal fluid (CSF) study, complete blood count, liver function tests, urinalysis and blood chemistry before and upon conclusion of treatment.

Based on reports so far published, the dosage of praziquantel used was that established as adequate: $50 \mathrm{mg} / \mathrm{kg}$ body weight/day, divided into three doses. Since the purpose of the trial was to demonstrate the cysticidal effect of praziquantel in the various groups, we tried to avoid, as far as 


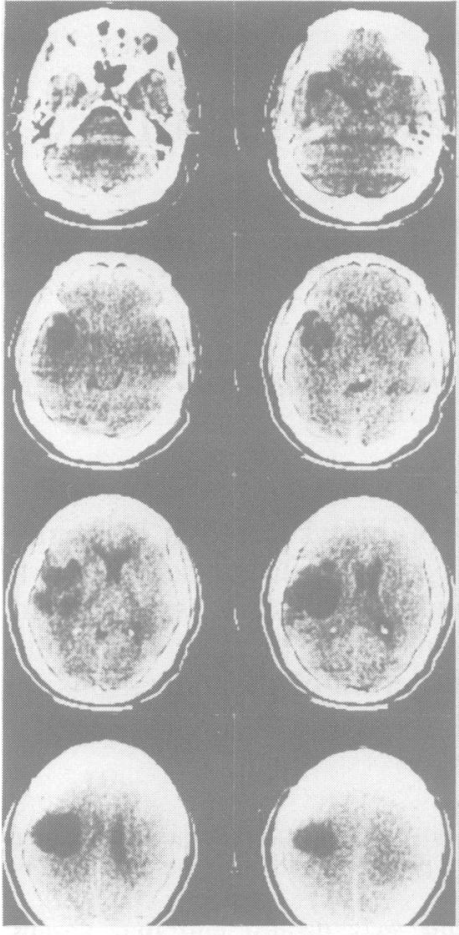

I

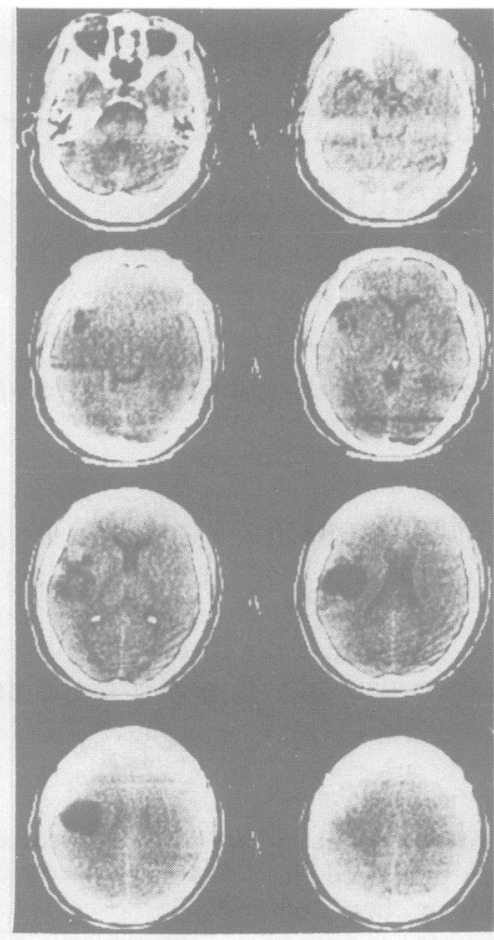

II

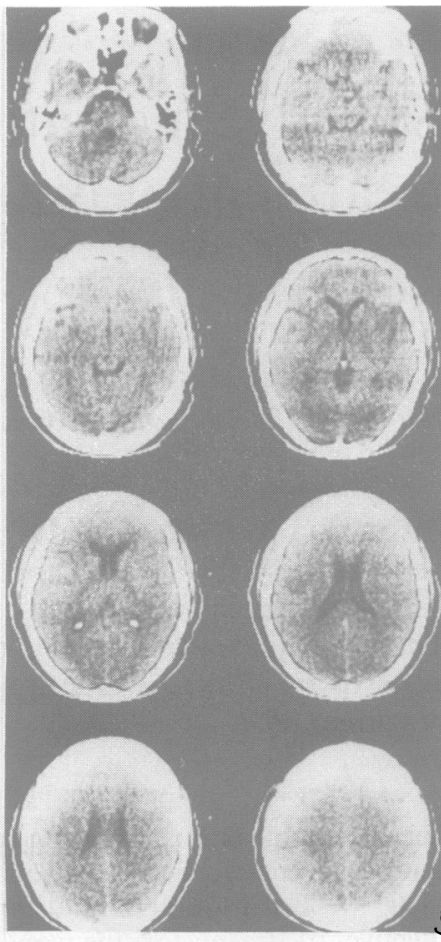

III

Fig 1 Group 1 patient: multiple cysticercous cysts of various sizes occupying the left temporal insula. Treatment initiated on 28 December 1982 (I); after 30 days of treatment all small cysts disappeared and the largest one shows signs of involution (II, 27 January 1983). The follow-up control examination performed after 7 months shows a ghost image in the site previously occupied by that cyst (III, 25 July 1983).

possible, using other drugs and/or applying surgical procedures which might have altered the value of the observations. For those patients who were under anticonvulsant or other systemic therapy before receiving praziquantel, the treatment regime was maintained.

In the first two cases, the initial CT was conducted one month after concluding 15 days of treatment. However, upon detecting that by then it was possible to observe changes in the CT scans suggesting effects of the drug on the cysts, it was decided to scan weekly for the next patients. From these observations it was determined in which cases it could be considered that the treatment has achieved its purpose after 15 days praziquantel intake and in which ones it should be extended to 30 days. Subsequent CT scans were continued for one year, monthly for the first 3 months, and every 3 months thereafter, until the 12-month follow-up was completed. In this manner, by combining criteria derived from the clinical neurological assessment and those from CT scanning, it was possible to establish the following:

1 If after 15 days treatment with the recommended dosage most cysts either disappeared or clearly showed involution signs on the later CT scan, treatment was stopped.

2 If, on the contrary, after the initial treatment time, several cysts persisted or did not clearly show involution signs, it was necessary to administer the drug for 30 days.

3 When, after a period of time, on subsequent CT scans the cysts had regained size and shape, praziquantel should be administered again for 15 days.

4 Neurosurgical procedures were considered necessary, either when complications inherent to the disease required it (for instance, in cases of hydrocephalus with signs of intracranial pressure rise) or because cysts had not shown changes caused by action of praziquantel and their presence became harmful for the patient.

\section{Results}

The sequence of CT scans clearly showed that the morphology of cysts underwent rapid changes: the size of vesicles began to diminish, their rounded and turgescent appearance was lost, their margins became blurred and the surrounding parenchymal attenuation became altered, resulting in changes known to be indicative of an inflammatory reaction. In most cases of Groups 1 and 2, this sequence became manifest as early as the first week of observation and progress was noted in cyst changes 3 to 4 weeks later when the smaller cysts disappeared. Larger ones took 


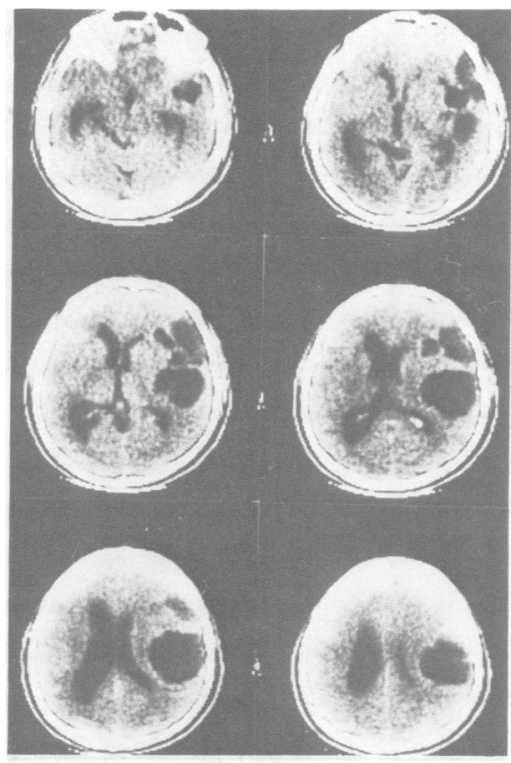

I

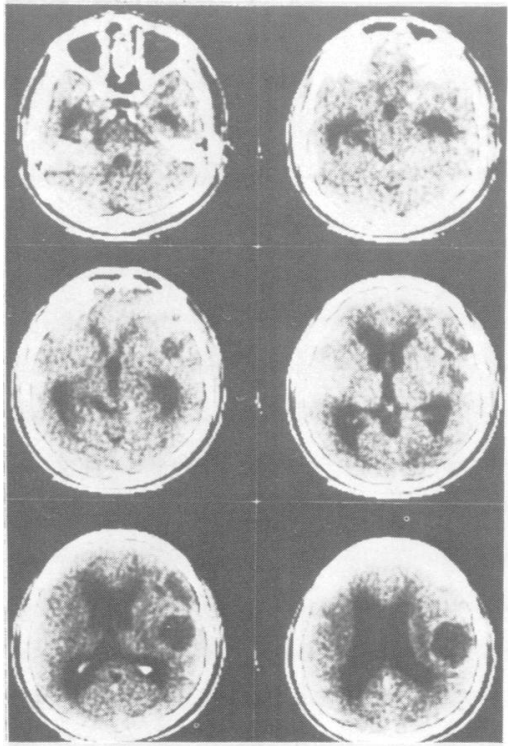

III

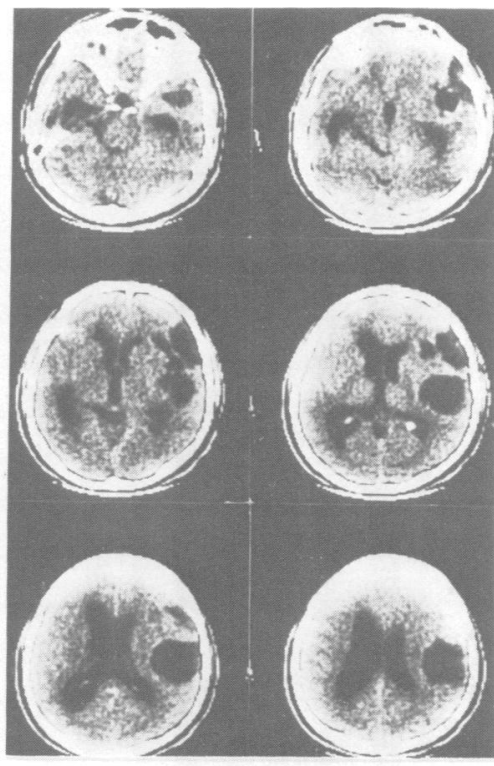

II

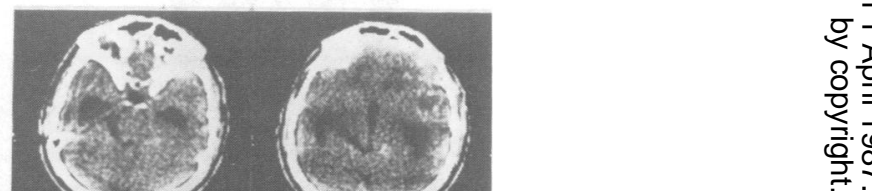

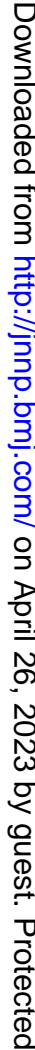

Fig 2 Group I patient: variously sized cysts in the right temporal region and ventricular system dilatation. Treatment was initiated on 11 November 1982. (I) and 30 days duration, because lesions showed a slow involution and did not completely disappear in the first follow-up CT (II 22 November 1982.) CT scan taken 5 days after conclusion of treatment shows disappearance of small cysts as well as involution of larger ones (III 27 December 1982.) CT scan 3 months after treatment stopped shows "ghost" where the largest cyst was. No increased hydrocephalus was noted (IV 25 March 1983). 


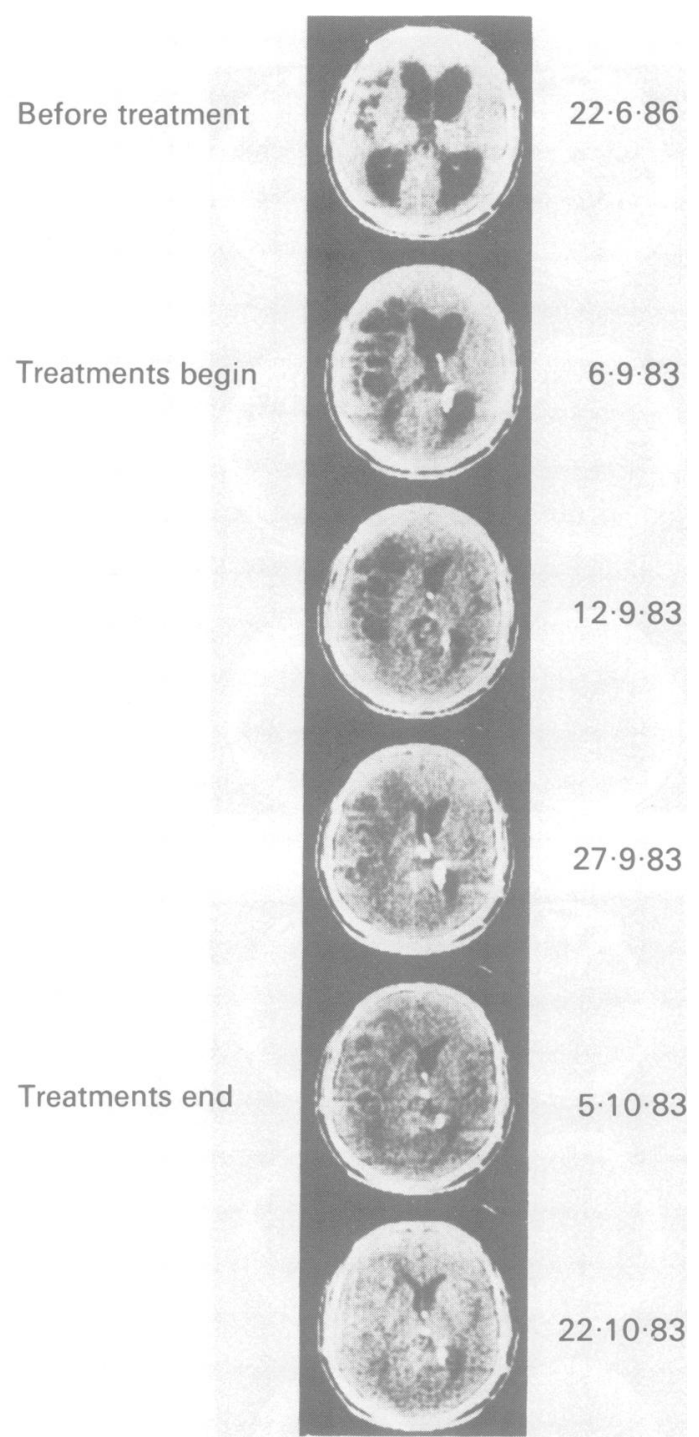

Fig 3 Group 2 patient showing numerous cysts in the left temporal insula. Before receiving praziquantel, this patient underwent ventriculoatriostomy for relief of hydrocephalus. The drug was administered for 30 days. Scan shows two catheters for ventriculoatrial derivation, that hydrocephalus was diminished and that all cysts have disappeared.

from 6 to 8 weeks to vanish completely. But in all of the patients comprising the first two groups no cysts could be seen after 4 months. Occasionally, it could be seen that, as a residual change, a "ghost" image appeared in the sites previously showing cysts, but after an indeterminate period of time these spots also

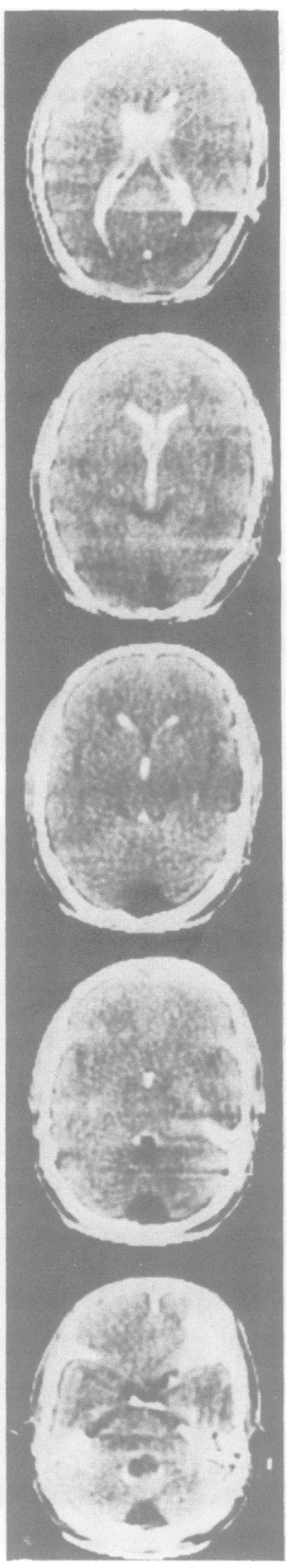

Fig 4 Ventriculography taken after injecting metrizamide into the shunt catheter. The ventricular system is clearly delineated and contrast medium reaches the fourth ventricle; here, the medium halts and clearly shows a bilobulatae cystic image occupying, distorting and increasing the size of the fourth ventricle. These are the features of intraventricular cysts. 

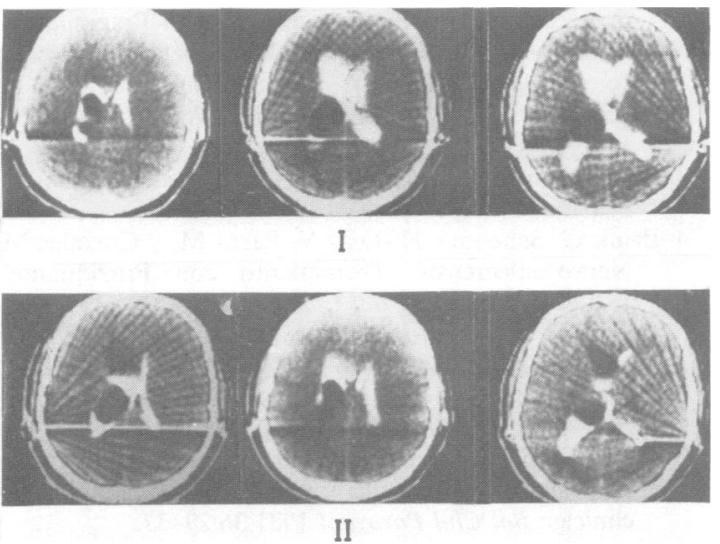

Fig 5 Cysticercous cysts shown in the left lateral corpora. Lesions did not show any changes of size or location after 30 days of treatment with praziquantel. (I before treatment; II after 30 days $P Z Q$ ).

tended to disappear (figs 1-3).

In one case which has been followed for 24 months, after 12 months it was noted that a higher density spot began to form at the site previously occupied by two large size cysts. For this reason, CT scans were continued every 3 months in this case. The spot became increasingly dense until it had the appearance of a calcified granuloma, which, since it is located at the site previously occupied by cysticerci, indicates that it is the residual lesion resulting from the killing and inactivation of the parasites. ${ }^{16}$

In three patients of Group 1, progressive dilatation of the ventricular system was noted, but only one of them developed intracranial hypertension, and, 4 months after completion of treatment with praziquantel, it became necessary to insert a ventriculoatrial shunt. In the other two patients, no increased dilatation of ventricular cavities was noted after one year of observation. Four months after completion of treatment, one patient died from status epilepticus as a result of withdrawing the anticonvulsants. Patients in Group 2, who because of hydrocephalus and intracranial hypertension, had undergone ventriculoatrial shunting before being treated with praziquantel, did not show any changes in the shape or size of ventricles upon disappearance of their cysts. Their clinical course has been stable.

Of patients in Group 3, four had cystic images occupying, dilating and distorting the fourth ventricle, and in one case cysts were located in the lateral ventricles (fig 4). The findings were obtained by injection of metrizamide into the shunt catheter, followed by CT. In none of these cases did the cystic images change, even after having received the drug for $\mathbf{3 0}$ days (fig 5). Four patients were operated upon and

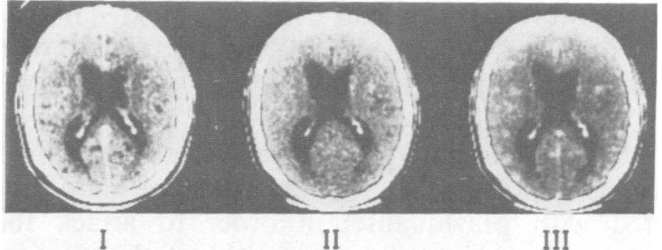

Fig 6 Miliary cysticercosis. Treatment initiated on 3 February 1982. (I). CT scans taken one year after treatment show no lesions when no contrast medium was used; after injecting contrast medium, the scan shows multiple hyperdense zones in the sites previously occupied by cysts, showing that as cysts involute, slowly progressing inflammation areas are formed (III, II January 1983).

one was not. The latter has maintained mild cerebellar symptomatology. Of the four patients operated upon, one died because of systemic complications; another, the patient with cysts located in the lateral ventricles, is currently in stable condition; the other two, with lesions into the fourth ventricle, have not responded satisfactorily.

The so-called "milliary cysticercosis" shows itself as small, disseminated, hyperdense spots, which may only be seen when CT scans are taken after intravenous injection of contrast medium. This feature was detected in the first patient of this group, and biopsies were taken of two lesions suitably located for this procedure. Histological examination revealed that they were cysticerci undergoing involution. After 15 days of treatment after biopsy, it was noted that other similar lesions did not show any changes: they only became visible as the contrast-medium study was performed, and continued doing so even on the final studies one year after the treatment ended. In the first case, some of the cystic images followed the evolution observed in the first two groups. In the remaining patients of this last group, hyperdense spots did not show any changes (fig 6).

Since the aim of this report is to establish the basis of the selective use of praziquantel, both side effects and the few nonspecific changes detected in the cerebrospinal fluid (CSF), as well as the lack of change in the biological constants studied, which have been previously reported elsewhere, have been omitted. ${ }^{11-16}$

\section{Conclusions}

Based on the experience gained after strict study of the cysticide effect of praziquantel on 40 patients with brain cysticercosis, selective criteria for the use of this drug may be established as follows:

1 Active cerebral cysticercosis, as characterised by the presence of cysts located in the brain parenchyma or the subarachnoid space, must be treated with praziquantel. 
2 Cerebral cysticercosis with cysts located only in the ventricular system should not be treated with praziquantel.

3 Mixed cysticercosis forms, that is, those with cysts both in the parenchyma and the subarachnoid space, as well as in the ventricular system, should be treated with praziquantel in order to attack the former, with subsequent assessment of the surgical indication for removing the latter.

4 Milliary cysticercosis should be treated with praziquantel, taking into account that it is not possible to state positively that those cases only have cysticerci undergoing involution. It is likely that there may also be either larvae or growing cysticerci which, owing to their size, may not be visible in the CT scans.

5 The method of examination both for diagnosing cysticercosis and assessing the indication, as well as the length of treatment with praziquantel, is serial computed cranial tomography.

6 The dosage of praziquantel is $50 \mathrm{mg} / \mathrm{kg} /$ day.

7 In most cases, the length of treatment should be 15 days. Computed cranial tomography scans, serially taken, shall enable one to decide if treatment should be extended to 30 days.

8 Patients showing very large or highly numerous cysts should be definitely treated for 30 days.

9 The need for neurosurgery should be assessed on an individual patient basis.

10 Use of any other medications such as analgesics, anticonvulsants, corticosteroids, or antihistamines, must be evaluated for each particular case, and they are to be administered as symptomatic agents in cases presenting side effects following the intake of praziquantel. Systemic treatment initiated before giving praziquantel must not be withdrawn.

This paper was presented at the Satellite Symposium on Neurocysticercosis in the Americas. XII World Congress of Neurology. Hamburg 1985.

\section{References}

1 Rim HJ, Won CR, Chu JW. Studies in human cys- ticercosis and its therapeutic trial with Praziquantel. (Embary 8440). Korea Univ Med J 1980;17:459-72.

2 Gómez JA, y Mejía A. Tratamiento de la neurocisticercosis con Praziquantel. Neurol Colombia 1980;5:503-9.

3 Spina-França A, y Nobrega JPS. Neurocisticercose e Praziquantel. Rev Paul Med 1980;95:34-6.

4 Brink G, Schenone H, Diaz V, Parra M, y Corrales M. Neurocisticercosis. Tratamiento con Praziquantel. Estudio preliminar. Bol Chil Parasitol 1980;35:66-7.

5 Botero D, y Castaño S. Cisticercosis. Tratamiento con Praziquantel. Tribuna Medica Colombia 1981;63:31-6.

6 Robles C. Tratamiento médico de la cisticercosis cerebral. Gac Méd Méx 1981;117:355-63.

7 Groll E. Cisticercosis humana y Praziquantel. Una apreciación panorámica de las primeras experiencias clínicas. Bol Chil Parasitol 1981;36:29-37.

8 Spina-França A, Nóbrega JPS, Livramento JA, y Machado LR. Administration of Praziquantel in neurocysticercosis. Tropenmed Parasitol 1982;33:1-4.

9 Botero D, y Castaño S. Treatment of cysticercosis with Praziquantel in Colombia. Am J Trop Med Hyg 1982;31:810-21.

10 De Rezende G, Groll E. Praziquantel en el mundo. Salud Publica Mex 1982;26:683-90.

11 Lombardo L, Vasconcelos D, Cruz-Segura H. Tratamiento de la cisticercosis con Praziquantel. Informe preliminar de diez casos. Gac Med Méx 1983; 119(1):17-22.

12 Spina-França A, Rezende de G. Alteraciones del líquidog cefalorraquideo con el Praziquantel. Salud Publica Mex 1982;26:633-6.

13 Frohberg H, Shulse E, y Schencking M. Toxicologica profile of PZQ, a new drug against cestode and schis tosome infection, as compared to some other schis-? tosomicides. Artzneimittelforsch 1981;31(1)3a:555-65.

14 Frohberg H. Propiedades farmacocinéticas, Farmacológicas y toxicológicas del PZQ. Salud Publica Mex 1982;26:605-24.

15 Leopold G, Ungethum W, Groll E, Diekmann HW, Nowak H, Wegner DHC. Clinical pharmacology in normal volunteers of Praziquantel, a new drug against schistosomes and cestode. An example of a complex study covering both tolerance and pharmacokinetics. Eur J Clin Pharmacol 1981;14:281-91.

16 Lombardo L, Vasconcelos D, Cruz-Segura H. Effects of Praziquantel on Cerebral Cysticercosis. Gac Med Mex (In press). 